\title{
Research on eddy current pulsed thermography for Squats in railway
}

\author{
Ling-fan Feng ${ }^{1}$, Jian-ping Peng ${ }^{1 *}$, Kang Zhang ${ }^{1}$, Jie Bai ${ }^{1}$, Xiao-rong Gao ${ }^{1}$ \\ ${ }^{1}$ School of Physical Science and Technology, Southwest Jiaotong University, Chengdu \\ 610031, PR China \\ *Corresponding author: adams.peng@switu.edu.cn
}

\begin{abstract}
The squats is one typical defect of rolling contact fatigue(RCF) in railway, emerging from the rail surface and propagating under external load, which is defined as one major danger source of rail. Eddy current pulsed thermography (ECPT), which combines the eddy current and thermography testing, is studied to inspect the squats through simulation and experimental way. One 3D finite element method (FEM) is designed to simulate the squats based on ECPT. And then the experimental verification and features extraction processing have been carried out. The results show that ECPT can be used to quantify the squats in railway.

Keywords: Eddy current pulsed thermography, squats
\end{abstract}

\section{Introduction}

In 1970, a type of rolling conact fatigue (RCF) crack was named as squat by the British Railways[1], which was neglected and later classified as one major danger source of crack. The squats occur on the surface of the track and may lead to track break in severe cases. Therefore, it is necessary to make predictive judgments of squats.

Non-destructive testing(NDT) methods used to detect include some acoustic, optics, electromagnetic method. Ultrasonic testing is the most common technique in acoustics. However, because the squat is close to the surface, which is located in the dead zone of ultrasound detection, it may cause misdetection and missed check, so ultrasonic detection is not suitable for detection of squats; Eddy current technology can detect small cracks though temperature field. Pulse eddy current technology(ECPT) and thermal imaging technology will be combined to detect squats. Then through temperature field received by infrared thermal camera, the location and depth of defects could be estimated approximately.

Daren Peng[2] uses the lock-in thermal technique with the $2 \mathrm{HZ}$ excitation source to detect micro cracks, the response signals at the defects field and no defects field are compared, but it was difficult to detect the size of the cracks only by the lock-in thermal technique. The rich feature of frequency of pulse excitation eddy currents components is used to put forward a spectral amplitude method[3]. Principal component analysis(PCA) is used to bring forward that the subsurface crack depth has an exponential relationship with the spectral amplitude. PCA and independent component analysis (ICA) are compared to verify these conclusions[4]: the parameters of artificial defects and natural defects can be extracted. The peak value also could be used for the index of open cracks. However, analysis of any specific cracks is barely mentioned.

Therefore, this article focuses on the thermal imaging characteristics of this specific crack---squats. it is organized as follows: Part I, The formation process of squats and principles of ECPT; Part II, Simulation and data analysis; Part III , Experimental platform setup and data processing; Part IV: Brief conclusions.

\section{Method}

\subsection{The formation process of squats}

During the running of the train, the force analysis of the contact between wheels and rails is shown in Fig. 1. There is a great longitudinal and lateral stress in the wheel-rail contact area. Therefore, the rail surface is very prone to cracking, and when the longitudinal force exceeds a certain value, cracks in another direction are initiated along the front of the contact area. Under the cyclic stress of the wheel and rail, the cracks will continue to expand along the original direction, eventually resulting in crossover. Then they extend $2 \sim 3 \mathrm{~mm}[5]$ to the inside of the rail head with $20 \sim 30$ degree, which is similar to the angle crack at this time. The cracks further expand, and it may turn into the direction of the parallel rail surface or continue to expand inside, forming a crack as shown in Fig. 2(a)[10], it's a crack like L shape, and this state may causes the rail surface depression (as shown in Fig.2(a).), or eventually cause the fracture of rail at the worst case[6].

Fig. 2(b) is a cross view of a rail squat, which is used to further understand the formation of squats. The first stage is the formation of angle cracks, and the second stage, it will develop into a L-type cracks or deeper oblique cracks under the periodic contact force. One 3-D finite element models are established to show two stages of development which is from angle crack (Figure 2(c)) to the L-shaped crack( Fig. 2(d)) , and these two models are compared and analyzed. 
Driving direction

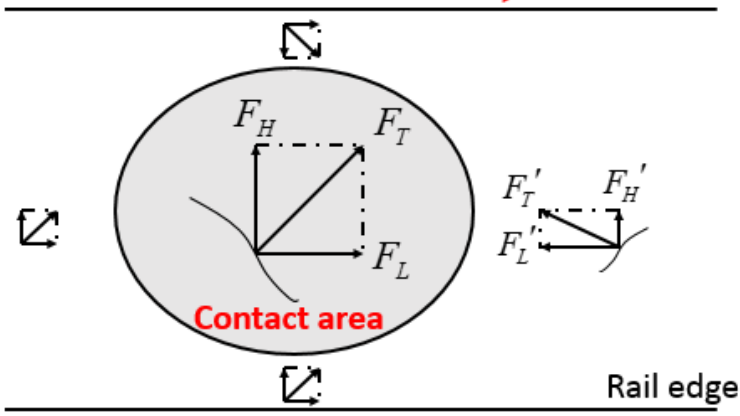

Fig. 1. Wheel and rail force analysis $\left(\mathrm{F}_{L}:\right.$ Longitudinal force: $\mathrm{F}_{H}$ Horizontal force $\mathrm{F}_{T}:$ Together force)

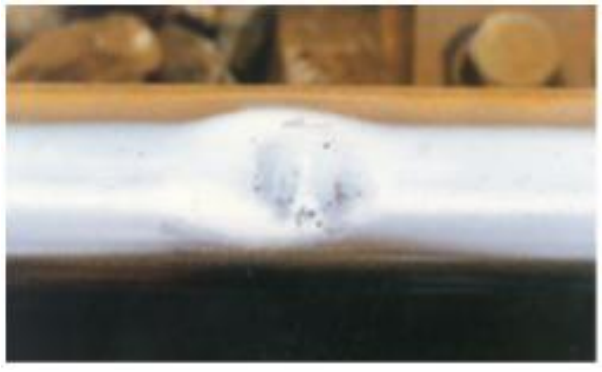

(a) rail squat

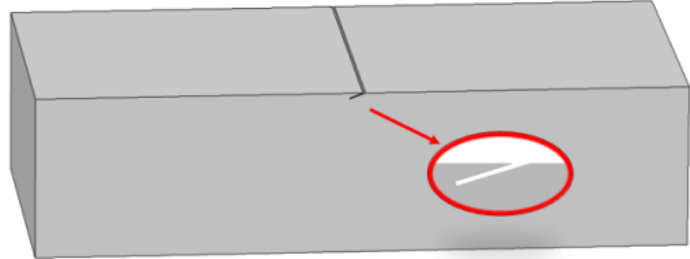

(c) Angle crack model

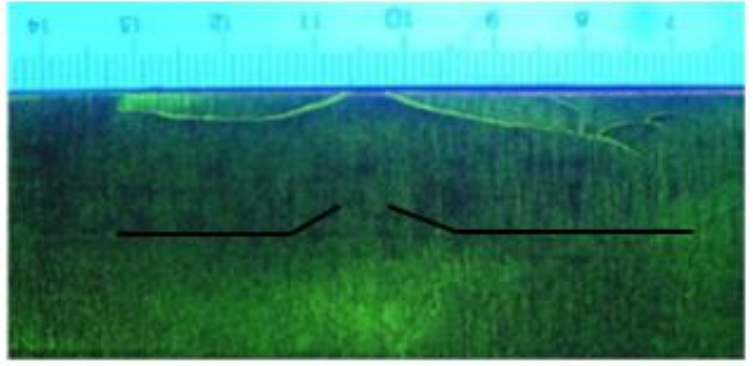

(b) Cross section for rail squat

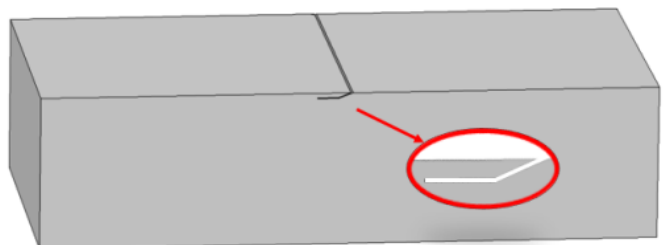

(d) L-shaped crack model

Fig.2. Model of two development stages

\subsection{Principle of ECPT}

Fig. 3 is a schematic diagram of eddy current pulsed thermography. When an alternating current(AC) excitation source is applied to excite the coil, magnetic induction line are induced around the wire according to the principle of electromagnetic induction. When the inductive line passes through the surface of the conductor, eddy current is formed on the surface of the conductor. The current produces Joule heating. When there is a crack on the surface or subsurface of the material, it disturbs the eddy current and the Joule heat distribution[7]. This disturbance is received by the infrared thermal camera.

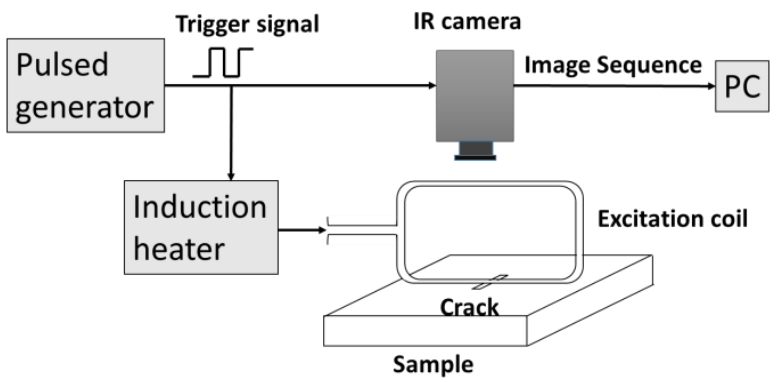

Fig.3. Experimental schematic

When the pulse eddy current excitation is a rectangular wave. As shown in equation (1), the excitation can be expressed as[8]: 


$$
f(t)=\left\{\begin{array}{c}
A, n T \leq t<(n+1 / 2) T \\
0,(n+1 / 2) T \leq t<(n+1) T
\end{array}\right.
$$

After the signal is transformed by Fourier transform equation, the resulting wave can be expressed like this:

$$
F_{0}(\omega)=A \tau \cdot S a\left(\frac{\omega \tau}{2}\right) \approx \frac{2 A}{\pi} \sin \omega t+\frac{2 A}{3 \pi} \sin 3 \omega t+\frac{2 A}{5 \pi} \sin 5 \omega t+\ldots
$$

Therefore, the rectangular wave can be decomposed into a superposition of many frequency signals in the frequency domain, and different depths of cracks respond differently to waves of these frequencies. Based on that, the pulsed eddy current can detect cracks of different depths in a large area simultaneously[9].

In a stable system, the heat transfer in an infinite area can be described as follows:

$$
\frac{\partial^{2} T}{\partial z^{2}}-\frac{\rho C}{k} \frac{\partial T}{\partial t}=0
$$

Convection heat in conductors:

$$
q_{c}=A h_{c}\left(T_{s}-T_{\infty}\right)
$$

$h_{c}$ is the heat transfer coefficient, $T_{s}$ is the surface temperature, $T_{\infty}$ the ambient temperature. Thesis rigorously discusses the relationship between the defect depth and the skin depth of the material, qualitatively gives the detectable depth of the eddy current detection of different materials, according to Joule's law. Due to the internal resistance of the material, the generated thermal power is:

$$
P_{w}=\frac{1}{\sigma}\left|J_{e}\right|^{2}=\frac{1}{\sigma}|\sigma E|^{2}
$$

This is the process of the temperature field that will be analyzed

\section{Analysis of simulation}

\subsection{Simulation setup}

The models as shown in Figure 2 (c), (d) are established using COMSOL 5.2 , the material parameters are shown in Table 1. The tested sample is considered as $80 \mathrm{~mm} \times 40 \mathrm{~mm} \times 20 \mathrm{~mm}$. A cylindrical wire was placed with a radius of 3.5 $\mathrm{mm}$, a length of $80 \mathrm{~mm}$, and a wire lift of $4 \mathrm{~mm}$, in the direction of vertical cracks. The coordinates of the two models are exactly the same, both angle of the crack and the rail surface are 20 degree, the length of the angle crack is set to $1.7 \mathrm{~mm}$, the transverse length in the $L$ crack is $2.7 \mathrm{~mm}$, and the depth value of $0.6 \mathrm{~mm}$.

The simulation process is divided into heating stage and cooling stage. The excitation current is $350 \mathrm{~A}$, the frequency is $250 \mathrm{KHz}, 200 \mathrm{~ms}$ heating time and $1000 \mathrm{~ms}$ cooling time are set, and the step length is 5 ms, which means that the collection frequency is $200 \mathrm{~Hz}$.

Table 1. Material parameters

\begin{tabular}{|l|l|}
\hline Parameters & Iron \\
\hline Relative permeability & 60 \\
\hline Electrical conductivity $(\mathrm{S} / \mathrm{m})$ & $4.68 \mathrm{e} 6$ \\
\hline Heat capacity $(\mathrm{J} /(\mathrm{kg} \cdot \mathrm{K}))$ & 475 \\
\hline Density $\left(\mathrm{kg} / \mathrm{m}^{3}\right)$ & 7850 \\
\hline Thermal conductivity $(\mathrm{W} /(\mathrm{m} \cdot \mathrm{K}))$ & 44.5 \\
\hline
\end{tabular}

Maximum heating temperature is extracted at 200ms in Fig. 4(a)/(b). Frames are also extracted at 400ms at cooling stag in Fig. 4(c)/(d), they both analyze the comparison of angle crack and L crack.

Fig.4 (a) is a typical thermal image for angle crack[10], which shows the spatial thermal characteristics of the angle crack.Fig. 4(b) depicts crack surface heat distribution image of L-type crack and angle crack, whose size are consistent with Fig. 2. Compared with Fig. 4(a), it can be observed that the temperature of the L-type crack tip is higher than that of the same-size angle crack, the temperature difference in the simulation reaches 20 degC. At the same time, the L-shaped crack has a larger area and higher temperature area on the side of the defect. Figure 4(c)(d) shows that the heat is diffused except the tip during the cooling phase, and the temperature of L-type crack drops slowly during the cooling process. The reason is that there is a transverse crack parallel to the excitation surface in the $L$ crack. This transverse part of the defect blocks the heat transfer from the surface in the cooling stage, so that heat flux is limited to this bounded area. Therefore, the higher temperature occurs at the corner region of the angled slot and area of transverse crack. Thus, the defect can be identified from a characteristic heat distribution in the thermal image/video captured by an infrared camera. 


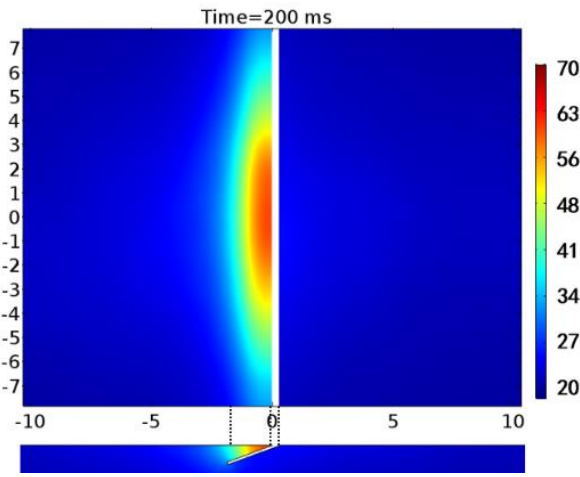

(a) angle crack at $200 \mathrm{~ms}$

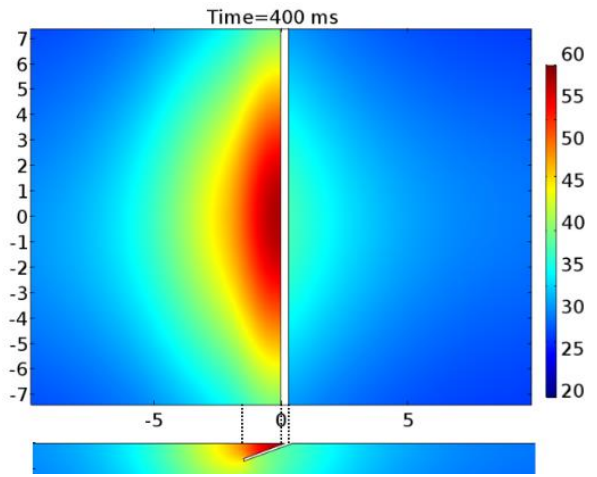

(c) angle crack at $400 \mathrm{~ms}$

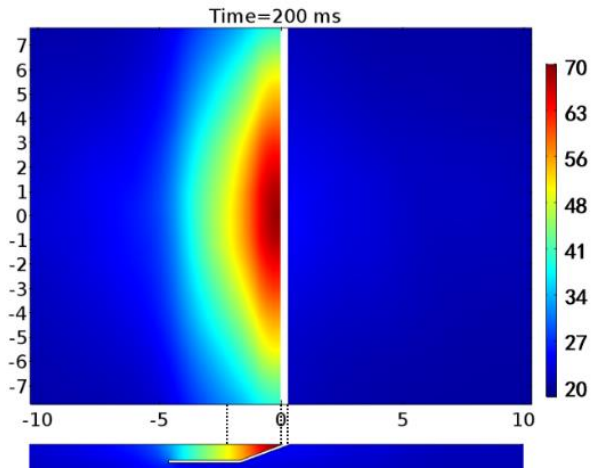

(b) L crack at $200 \mathrm{~ms}$

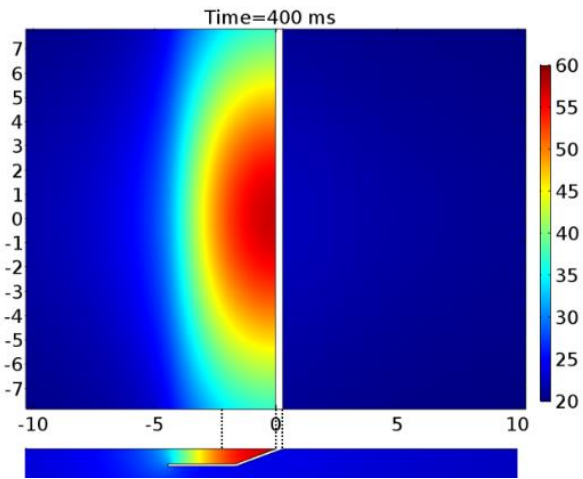

(d) L crack at 400ms

Fig.4. Simulation results of surface heat distribution of angle crack and L crack

A line located below the wire and in the vertical crack direction was extracted for analysis. Fig. 5(a) shows the temperature profile of the two lines respectively. Fig.5(b) shows the differential temperature between the $L$ and the angle cracks. It can be seen that the surface temperature near the $L$ crack are all higher than the oblique cracks. Compared with Fig.3, conclusions could be drawn as follows: The heat has not been transferred to the transverse region at the time of $200 \mathrm{~ms}$, but the existence of lateral regions makes that the accumulated heat at the $L$ tip is higher than the oblique cracks, and the heat around the transverse cracks is formed by thermal diffusion during cooling stage.

In the cooling stage of $400 \sim 500 \mathrm{~ms}$, the temperature at the tip falls rapidly, but the overall temperature in the large crack region remains high, and the gradient of the temperature distribution shows a slowly changing trend, which further validates the above conclusion.

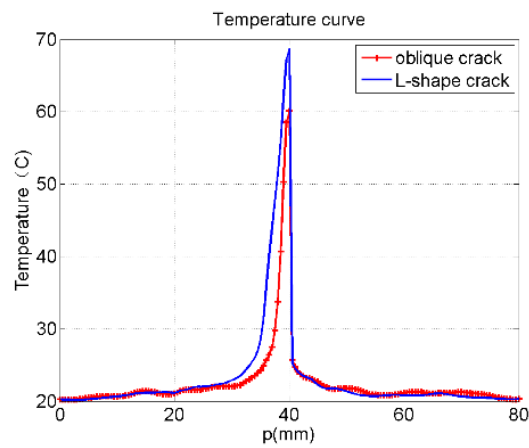

(a) Temperature diagram

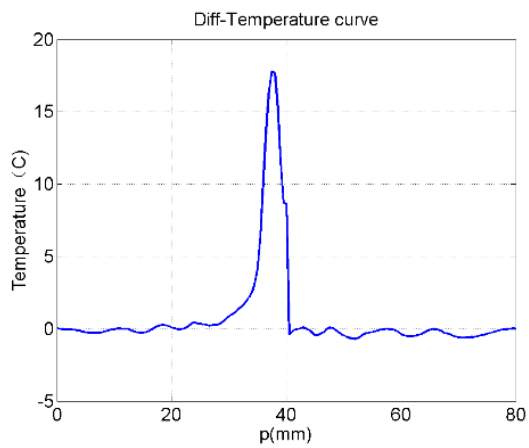

(b) Differential temperature

Fig. 5. Comparison between L crack and oblique crack

In this manner, the heating pattern for an angled defect with $L$ shape, which includes high temperatures at the slot side and low temperatures at the opposing side with a maximum temperature at the slot corner. Further more, thermal gradients at $L$ crack area keep small value, which can be used to index the $L$ crack's shape. 


\subsection{Isotherm analysis methods and results}

Fig. 6 is temperature distribution of angle crack and $L$ crack at $200 \mathrm{~ms}$, isothermal closed curves divides the temperature field. Fig. 6 (b) shows that the high temperature area of the $L$ crack area is bigger than the oblique crack's. The density of the isothermal line changes at $-2 \mathrm{~mm}$. This position happens to be the corner of the $L$ crack. Therefore, the center of the defect can be resolved by the gradient, which provides the basis for the analysis of the image characteristics and position of the later stage.

The gradient formula is:

$$
|G|=|\operatorname{grad}(u)|=\sqrt{\left(\frac{\partial u}{\partial x}\right)^{2}+\left(\frac{\partial u}{\partial y}\right)^{2}}
$$

Formulas written in vector form can be expressed as:

$$
\operatorname{grad}(u)=\frac{\partial u}{\partial x} i+\frac{\partial u}{\partial y} j
$$

It can be seen from Fig. 6 that the temperature distribution of L-crack in region A is similar to those in angle crack. In $\mathrm{L}$ crack model, along the crack direction, the gradient of the temperature curve changes, and the constant temperature tends to be stretched in the crack propagation direction. The temperature continues to drop until it is the same temperature as the non-defective area.

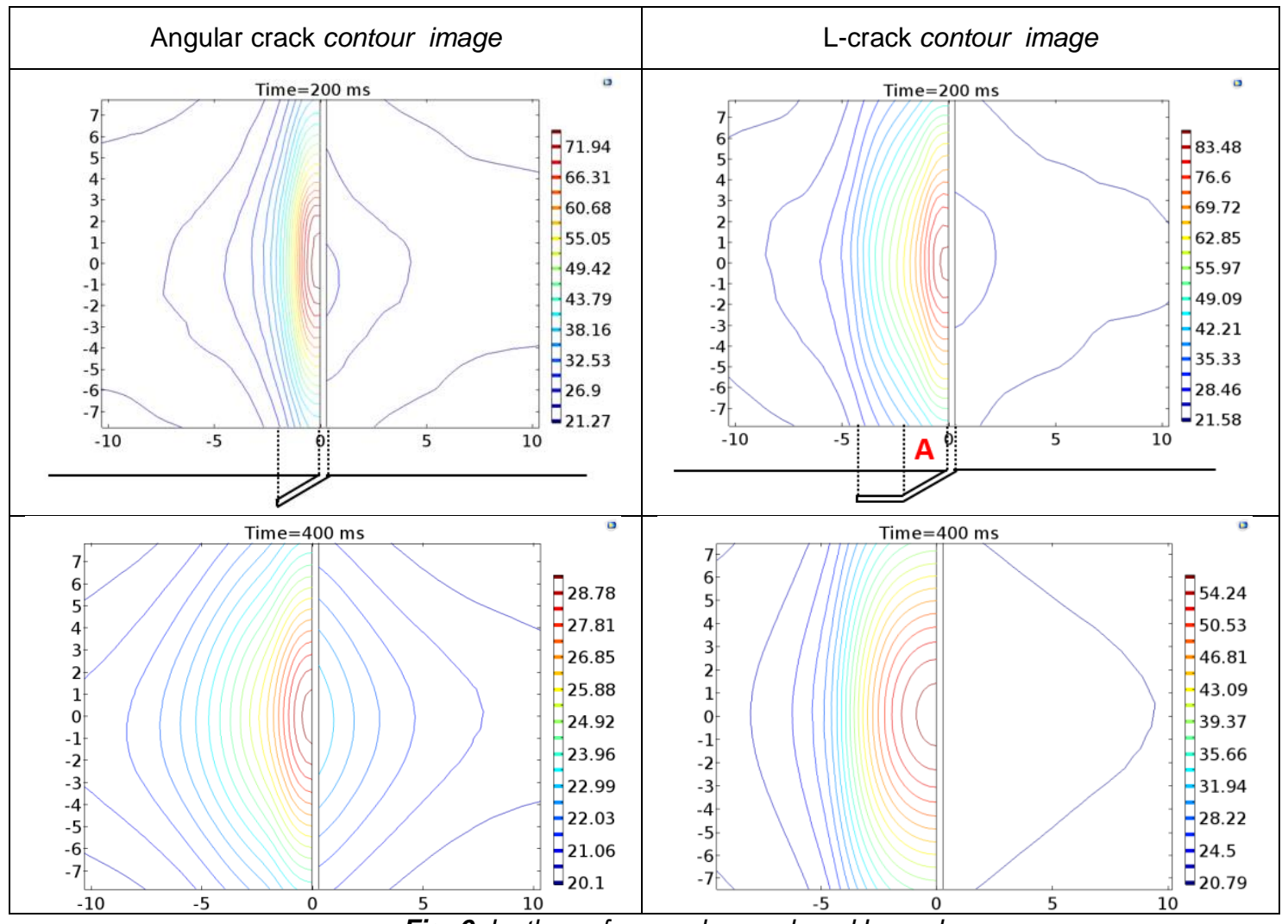

Fig. 6. Isotherm for angular crack and L-crack

\section{Analysis of experimental data}

\subsection{Experiment Setup}

As shown in Fig.7, the experimental system consists of an infrared camera, an induction heating device, a PC, and matching image acquisition software.

In this experiment, the excitation signal generated by the induction heater (Easyheat 224 from Cheltenham Induction Heating) had the frequency of $200 \mathrm{kHz}$ and the current of 350 Arms. The IR camera (FLIR SC655sc) had an uncooled detector with the maximum frame size of $640 \times 120$ pixels on a $7.5-14.0 \mathrm{~mm}$ sensor with the sensitivity of $\leq 50$ $\mathrm{mK}$. The infrared camera frame rate is $200 \mathrm{fps}$, the heating time is $0 \sim 200 \mathrm{~ms}$, the recording system has a $200 \mathrm{~ms}$ delay, and the total recording time is 2 seconds. The above experimental parameters are consistent with the simulation parameters. In the experiment, oblique cracks and $L$ cracks were processed on a test piece, and the sample was heated with a long straight wire to facilitate simultaneous observation. 


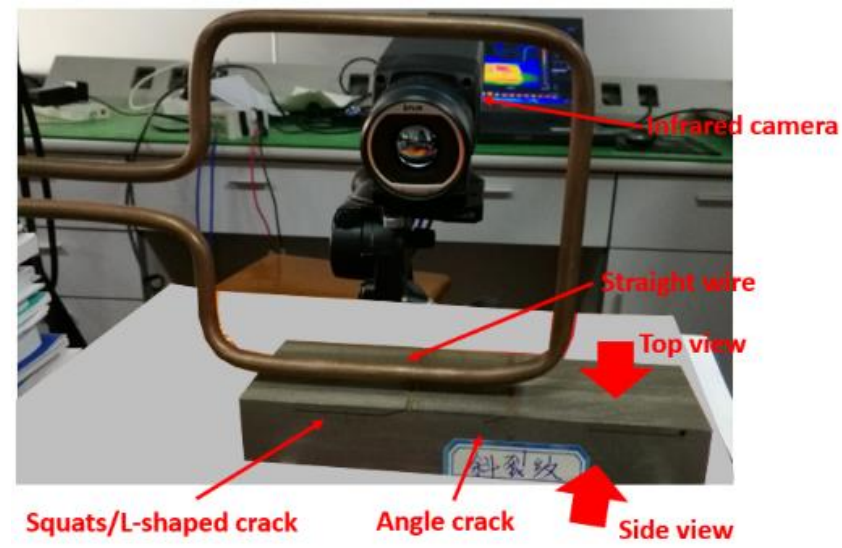

Fig. 7. Experimental setup

\subsection{Experiment of $L$ crack and oblique crack}

The observation angle and area of the thermal imager are adjusted to cover the upper surface and side areas of sample, which helps to observe the surface heating and thermal diffusion process at the same time, the thermal diffusion process inside the crack can also be indirectly observed through the side Image observation. As shown in Fig. 8, the time corresponding to (a), (b) is in the heating phase, and (c) (d) is in the cooling phase.

It can be seen that during the heating process, the Joule heat generated by the thermal energy is affected by the angle cracks, high temperature areas are concentrated near the tip of the two cracks, the defect side shows a high temperature distribution, non-crack side temperature is low, which are consistent with the simulation results .

From the side view, as is shown in Fig. 8(b), it can be observed that the temperature of inside is relatively different from the surface in the heating stage, which helps to clearly observed distribution and expansion of the crack. This is mainly due to the diffusion of heat to the lateral end regions. Following Fourier's thermal law, $L$ crack has a lower temperature at the distal end of the crack and is less affected.

In the cooling phase, heat conduction is the main role. Thermal energy from the high temperature region and the crack tip region of the surface diffuses into the inside of the tested block, and the shape of the crack is further clearly seen through the side view. Similar to the analysis in the simulation part, the side part of the crack prevents the thermal energy from further diffusing to the inside, accumulating in the side wall area and forms a high temperature distribution, it improves the visibility of the boundary profile of the crack. Compared with the angle crack and $L$ crack, the heat loss of the angle crack during the cooling period is very fast, and the temperature of the $L$ crack region is generally high. This conclusion is completely consistent with the simulation.

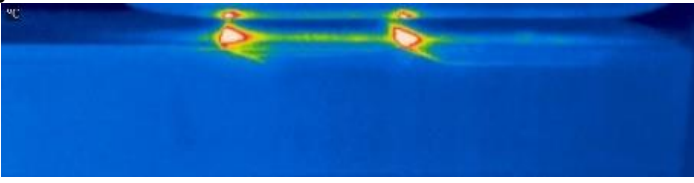

(a) $100 m s$

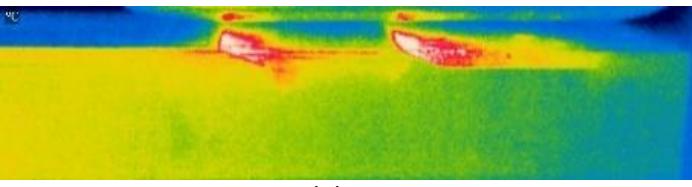

(c) $900 \mathrm{~ms}$

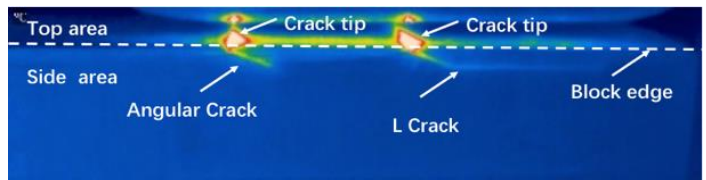

(b)200ms

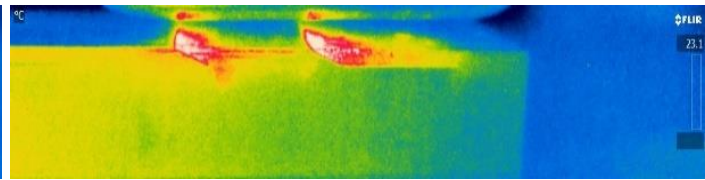

(d)2s

Fig.8. thermal image for top view and side view with artificial defect block

The change of the temperature of the two cracks' tip was extracted, and the temperature transient curve is shown in Fig.9. According to the basic working principle of the electromagnetic eddy thermal imaging, the angle cracks and Lcrack are exactly the same in the heating stage of the crack, and the influence on the surface vortex distribution tends to be consistent. Therefore, in the initial heating stage, the temperature field distribution is mainly affected by the eddy current distribution. The temperature trend of the two cracks is basically the same. 


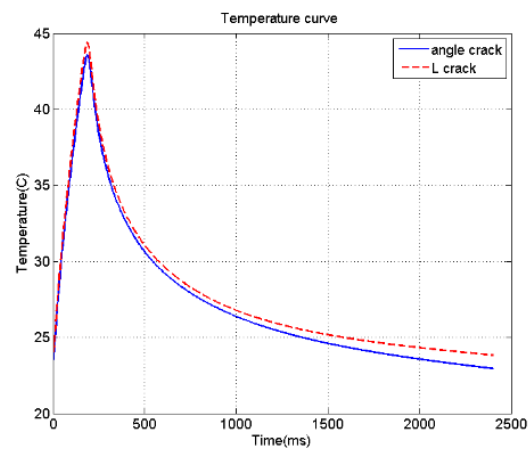

Fig.9. Two crack tip temperature comparison

\subsection{Experiment of real nature squat crack in rail head}

As is shown in Figure 10(a), crack1 2, crack2 is very close to crack1, its thermal imaging may be affected by crack1, as shown in Figure 10(b), it is a crack profile. Long straight wire heating is still used to heat the tested block, heating period is $200 \mathrm{~ms}$.

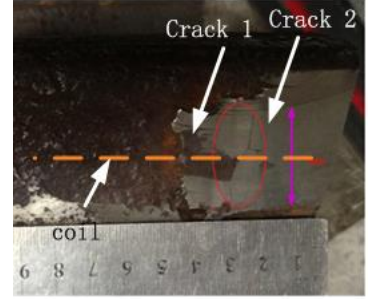

(a) Rail cracks

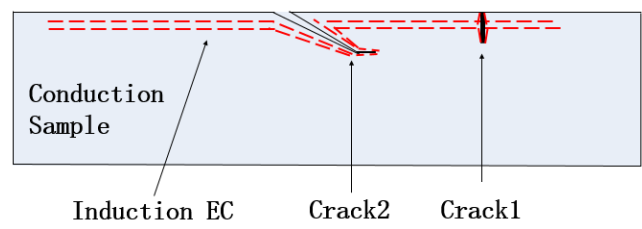

(b) Crack profile

Fig. 10. Crack status

As Fig. 11(a) shown, the thermal images extracted at 200ms and $400 \mathrm{~ms}$. The spatial thermal features as higher temperature at angular crack side is observed, which keeps the same as the simuliation results. At cooling stage of $400 \mathrm{~ms}$, the shape of real crack can be defined through the high temperature boundary. Fig. 11(b) provides spatial temperature distribution using the line scan result along the excitation coil, the big temperature gradient appears at the crack tip and crack boundary. Meanwhile, one small vertical crack also be oobserved at position 350 .

The oblique and vertical cracks are identified by the thermal features shown though Figure 11(c). It can also be verified that the smaller the crack angle is, the more heat accumulated at the crack tip. These two different thermal characteristics reflect the judgment of different crack types.

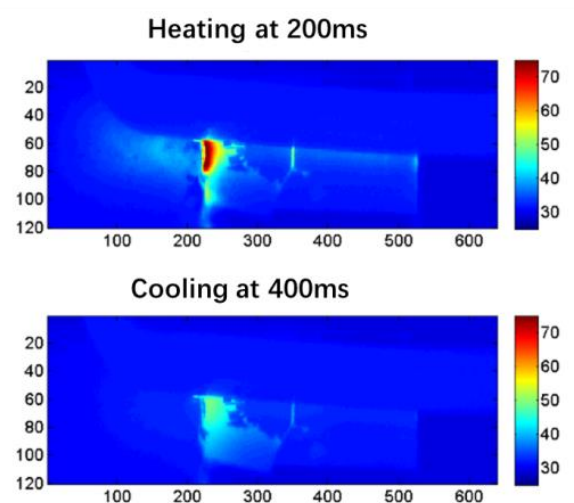

(a) thermal image at heating and cooling

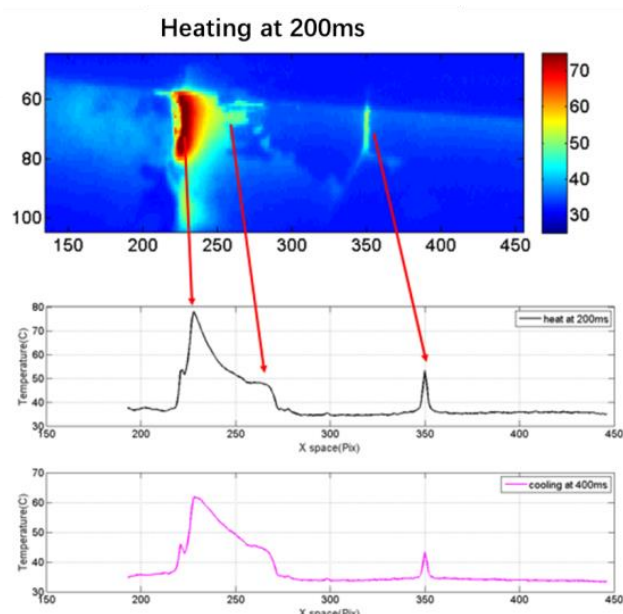

(b) thermal spatial features

Fig. 11. Thermal image for rail squat 


\section{Conclusion}

In this paper, according to the typical formation process of the squat in the rail fatigue defect, the squat is divided into two stages: angle crack and L crack. The Finite element model(FEM) model of the squats is established based on the difference of angle crack and $L$ crack. Through the relevant simulation and experimental work, the following conclusions can be drawn:

(1) Comparing the simulation results with the experimental results, it shows that the L-shaped crack defect model can effectively be used to simulate the form of squats in rail.

(2) Through the analysis, the intrinsic relationship between the geometry of the squat and the distribution of the surface temperature field over time is studied. The lateral region effectively blocks the heat diffusion, making the overall temperature of the $\mathrm{L}$ crack higher; the lateral region of squat has almost no heat accumulation in the induction heating stage, and the heat is mainly caused by heat conduction.

(3) The development stage of crack can be identified by the thermal imaging and the spatial temperature distribution characteristics. That means the approximate crack shape at this time can be judged. In further work, the rail occultation will be studied to determine the crack shape, The problem of reconstructing and quantifying the combination of flaws in rails are expected to be resolved.

\section{Acknowledgement}

This work was supported in part by the National Natural Science Foundation of China under Grant 61771409

\section{REFERENCES}

[1] Grassie S L. Squats and squat-type defects in rails: The understanding to date[J]. Proceedings of the Institution of Mechanical Engineers Part F Journal of Rail \& Rapid Transit, 2012, 226(3):235-242.

[2] Peng D, Jones R. NDI of Rail Squats and Estimating Defect Size and Location Using Lock-In Thermography[J]. Engineering, 2013, 5(4):29-38.

[3] Zhou Deqiang, Tian Guiyun, You Lihua. Pulse eddy current defect detection based on spectrum analysis[J]. Journal of Instrumentation, 2011, 32(9): 1948-1953.

[4] Yang J, Cheng Q. A comparative study of independent component analysis with principal component analysis in geological objects identification. Part II: A case study of Pinghe District, Fujian, China[J]. Journal of Geochemical Exploration, 2015, 149:136-146.

[5] Li X, Xi W., Study on the Formation Mechanism and Prediction of Rail Tread Squats Based on Load Factor and Traction Coefficient [J]. China Railway Science, 2012, 33(b08):12-16.

[6] Farjoo M, Daniel W, Meehan P A. Modelling a squat form crack on a rail laid on an elastic foundation[J]. Engineering Fracture Mechanics, 2012, 85(85):47-58.

[7] Wysockafotek O, Maj M, Oliferuk W. Use Of Pulsed IR Thermography For Determination Of Size And Depth Of Subsurface Defect Taking Into Account The Shape Of Its Cross-Section Area[J]. Archives of Metallurgy \& Materials, 2015, 60(2):615-620.

[8] Newman G A, Hohmann G W, Anderson W L. Transient electromagnetic response of a three-dimensional body in a layered Earth[J]. Geophysics, 2012, 51(8):1608-1627.

[9] Wilson J, Tian G Y, Abidin I Z, et al. Pulsed eddy current thermography: system development and evaluation[J]. Insight: Non-Destructive Testing \& Condition Monitoring, 2010, 52(2):87-90.

[10] F. R. A. U.S. Department of Transportation, "Rolling Contact Fatigue: A Comprehensive Review," 2011. 